\title{
Handheld Real-time Volumetric 3-D Gamma-ray Imaging
}

\author{
Andrew Haefner ${ }^{\mathrm{a}}$, Ross Barnowski ${ }^{\mathrm{b}}$, Paul Luke ${ }^{\mathrm{a}}$, Mark Amman ${ }^{\mathrm{a}}$, Kai Vetter ${ }^{\mathrm{b}, \mathrm{a}}$ \\ ${ }^{a}$ Lawrence Berkeley National Lab - Applied Nuclear Physics, 1 Cyclotron Road, Berkeley \\ CA, 94720, United States of America \\ ${ }^{b}$ Department of Nuclear Engineering, UC Berkeley, 4155 Etcheverry Hall, MC 1730, \\ Berkeley CA 94720, United States of America
}

\begin{abstract}
This paper presents the concept of real-time fusion of gamma-ray imaging and visual scene data for a hand-held mobile Compton imaging system in 3-D. The ability to obtain and integrate both gamma-ray and scene data from a mobile platform enables improved capabilities in the localization and mapping of radioactive materials. This not only enhances the ability to localize these materials, it provides important contextual information of the scene, which once acquired, can be reviewed and further analyzed subsequently. To demonstrate these concepts, the High-Efficiency Multimode Imager (HEMI) is used in a hand-portable implementation in combination with a Microsoft Kinect sensor. This sensor, in conjunction with open-source software, provides the ability to create a 3-D model of the scene and to track the position and orientation of HEMI in real-time. By combining the gamma-ray data and visual data, accurate 3-D maps of gamma-ray sources are produced in real-time. This approach is extended to map the location of radioactive materials within objects with unknown geometry.
\end{abstract}

Keywords: Compton imaging, Gamma-ray imaging, SLAM, 3-D Imaging, Volumetric Imaging, Data Fusion

Preprint submitted to Nuclear Instruments and Methods in Physics Research ANovember 19, 2016

(C) 2016. This manuscript version is made available under the Elsevier user license http://www.elsevier.com/open-access/userlicense/1.0/ 


\section{Introduction and Background}

Detecting, localizing, and mapping gamma-ray emitting objects in real-world environments are important capabilities for many applications including nuclear security and safety, emergency response, consequence management, and nuclear contamination remediation. Currently, static or portable non-imaging detectors and in some cases, static gamma-ray imaging instruments are employed, depending on the specific requirements. Combining hand-portable gamma-ray imaging systems with contextual sensors such as cameras or depth sensors enables the reconstruction of a scene with the embedded gamma-ray emission distribution in three dimensions in real-time. The ability to localize radioactive materials in arbitrary environments in three dimensions provides important additional details about their spatial distribution, including contextual information; for example, the relation of the materials (inside or outside) to other objects in the scene. Since the scene with integrated gamma-ray image information, the path of the system through the scene, and the associated time evolution can be stored digitally, all the information can be re-analyzed after the measurement is complete. In this way, the measurement can be replayed and other clues and features can be studied. Scene-data fusion can also provide more sophisticated capabilities in areas with limited or no access, such as inside of shipping containers, providing new means of localizing and characterizing radioactive materials within such containers. This method also improves capabilities for search scenarios by helping to mitigate the geometric inverse distance square intensity reduction as the portable imager can be brought closer to the detected emission source. The ability to move freely throughout a scene and to observe multiple perspectives of an object can further aid in the detection and characterization of sources and associated shielding. Additionally, the three-dimensional (3D) scene model can be used to constrain the position of gamma-ray emitting objects, potentially reducing computational time and improving accuracy of the source reconstruction.

Static and two-dimensional gamma-ray imaging in combination with two- 
dimensional overlays on visual images is available commercially [1][2], and has been demonstrated in relevant environments, such as contaminated areas in Fukushima Prefecture, Japan [3]. Near-field 3-D gamma-ray imaging on the order of millimeters has been demonstrated with a handheld Compton camera [4]. In larger areas, 3-D gamma-ray imaging was demonstrated with a coded aperture system combined with a LIDAR scan using a static measurement [5], which is limited due to the single perspective. Volumetric 3-D imaging was demonstrated in offline processing with multiple perspectives combined with a LIDAR scan and Compton imaging [6]. More recently, the concept of real-time volumetric imaging and scene data fusion was demonstrated employing a cart-based Compton imaging instrument consisting of three-dimensional position sensitive HPGe detectors paired with a Microsoft Kinect system [7]. In that configuration, the size and weight of the cart limited the types of measurements that were possible. Nevertheless, real-time 3-D scene data fusion was successfully demonstrated with that system. This paper presents an implementation of realtime 3D scene data fusion on a hand-portable instrument and demonstrates the effective localization of a range of gamma-ray sources around or within objects. Several of the measurements shown would not be possible from the cart based system.

Our approach in this paper is to perform near real-time (i.e. within seconds) 3-D gamma-ray reconstruction of scenes from a handheld system. As the system collects data, a 3-D estimate of the source distribution is created and updated as more data are collected. This paper introduces the measurement system, the data processing approach and demonstrates a variety of capabilities with relevant lab measurements. The lab measurements begin with single sources on open surfaces to convey the 3-D and real-time nature of the reconstruction. A second measurement serves to demonstrate the difference with conventional static 2-D imaging. Then the simultaneous measurement of multiple source energies and locations is shown. Finally, measurements of sources within objects are shown to demonstrate localization for passive object interrogation scenarios. While this paper focuses on our specific handheld implementation, the mobile 3D 
scene-data fusion concept can also be integrated with autonomous platforms on land or in the air. This has been demonstrated with the system used in this work in combination with contextual visual cameras on an Unmanned Aerial System (UAS) in the Fukushima Prefecture already [8]. In general, the advantage of such a system is the ability to deploy into areas with limited access or where the risk to human operators is too high. The advantage of the 3-D approach is to better account for the 3-D geometry of the scene. UAS deployments also enhance the effectiveness in the mapping of radioactive contamination [9]. The concept of 3-D scene data fusion can easily be extended to the detection and mapping of neutrons or other radiation, including imaging or non-imaging modalities.

\section{High Efficiency Multimode Imager and System}

The detector used for this work is the High Efficiency Multimode Imager (HEMI). The HEMI design consists of 96, $1 \mathrm{~cm}^{3} \mathrm{CdZnTe}(\mathrm{CZT})$ crystals, each with a coplanar grid (CPG) readout, arranged in a 2-plane active-mask configuration with the front plane half populated in a random mask pattern (32 detector elements) and the back-plane fully populated (64 detector elements) [10] [11]. The active mask configuration was selected to allow the simultaneous use of coded aperture and Compton imaging modalities. This work focuses on HEMI's Compton imaging capability. The use of CZT detectors operated at room-temperature with CPG readout and active coded-mask provides simple operation with an excellent performance-to-weight ratio. The total mass of the instrument including batteries for HEMI is about $3.6 \mathrm{~kg}$. The battery life for HEMI is about 5 hours, with the battery life of the laptop and tablet of several hours remaining the limiting factor for mobile measurements. HEMI is characterized by a relative energy resolution of $2.5 \%$ FWHM and an angular resolution of about $10^{\circ} \mathrm{FWHM}$ at $662 \mathrm{keV}[12]$. One benefit of this free moving approach is that moving HEMI close to objects serves to mitigate its coarse angular resolution because the spatial resolution depends on the distance to objects. This point is explored more in Section 4.1. 
HEMI is combined with the Microsoft Kinect sensor in a hand-portable implementation in this work as illustrated in Fig. 1. All of the associated com-

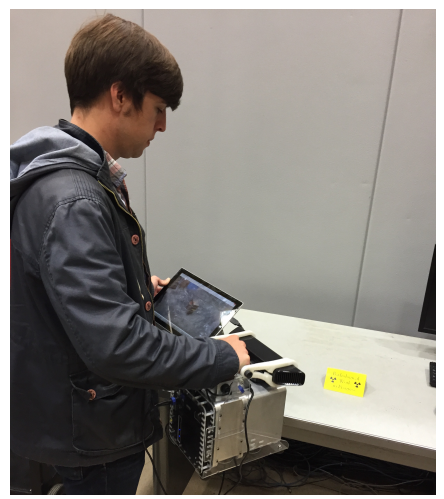

Figure 1: Hand-portable operation of the integrated HEMI and Kinect system. The user carries the device around the scene and receives actionable gamma-ray imaging feedback in real-time on the tablet.

puters for collecting data with HEMI are shown in Fig. 2 and include: a tablet for instrument control and data visualization, and a laptop that performs the visual data processing as shown in Fig. 2. The Kinect provides both RGB images and dense point cloud representation of the scene within its field-of-view of $43^{\circ}$ vertical and $57^{\circ}$ horizontal at up to $30 \mathrm{~Hz}$ frame rate. Using Simultaneous Localization and Mapping (SLAM) performed on the laptop, the visual and geometric data from the Kinect is processed to create a 3-D model of the environment and simultaneously track the device position and orientation in real-time. This work utilizes the RGBD-SLAM algorithm [13], which relies on the visual RGB information along with the depth information from the Kinect.

The visual reconstruction performed on the laptop utilizes a GPU to operate in real-time. The fusion of the gamma-ray image and visual data is performed on the tablet and is less computationally intensive compared to the SLAM component. The tablet is a Microsoft Surface 3, with 8 GB of RAM and a dual core i7 Intel processor. This is sufficient for real-time 3-D data fusion, which includes the combination of the $3-\mathrm{D}$ visual model and the gamma-ray 


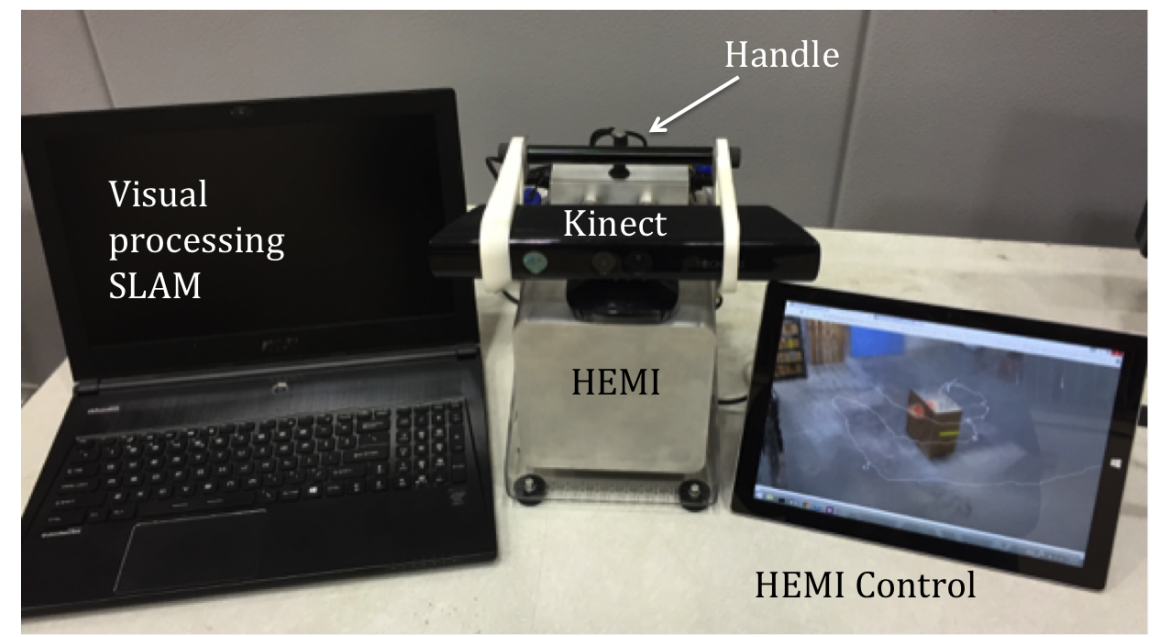

Figure 2: HEMI with Kinect and the computers used for real-time processing of data.

Compton imaging events, in areas the size of a single room. The computed visual model and detector locations are sent wirelessly to the tablet where the data are integrated with the gamma-ray data. Real-time feedback is displayed on the tablet as the data are collected and processed.

\section{Reconstruction Approach}

Compton imaging requires the reconstruction of the gamma-ray interaction positions within the detector array. The positions of the first two interactions define the symmetry axis of a cone whose opening angle is defined by the energy deposited in the first interaction and the energy of the incident gamma ray. The cone represents all possible incident directions for the specific gamma ray. The accumulation of multiple cones, each determined from an independent interaction sequence, is necessary to reconstruct the gamma-ray source distribution. In HEMI, the locations and energies of the gamma-ray interactions are determined by the positions of and measured energy in each of the CZT detector elements. Typically, an interaction sequence involving two elements is required, and the sum energy is assumed to be the incident gamma-ray energy. In this analysis, 
both possible scattering sequences from each pair of interactions are used in the gamma-ray image reconstruction. This decision is based on the assumption that given the low number of counts, losses of potential Compton cones due to mis-sequencing should be minimized. As an additional requirement in the Compton event reconstruction, a lever arm cut of $2.1 \mathrm{~cm}$ is applied, where the lever arm is computed as the distance from the center of each detector element in which that event occurred. This was used to remove events involving adjacent or diagonal detector elements. These events have very poor angular resolution due to the size and proximity of the detectors. The cone width is set to $10^{\circ}$ FWHM, reflecting the expected angular uncertainty of the HEMI system.

For the gamma-ray image reconstruction, a list mode maximum likelihood expectation maximization (LM-ML-EM) method was used [14]. Compton imaging often requires list-mode operation due to the high number of dimensions across the data space, which can include 3-D interaction positions and energies, and location and orientation of the detector, etc. ML-EM is known to have convergence issues for distributed sources [15] (for example, different image frequencies converge at different rates), but this work focuses on point or compact sources relevant for many search applications. There are three main computational components associated with ML-EM: computing the system matrix, computing sensitivity, and computing the iterations. Sensitivity is assumed to be uniform, which is reasonable for the point source scenarios investigated in this work. Distributed sources would require a more detailed sensitivity calculation.

One additional challenge with reconstructing arbitrary environments is the handling of cones that do not fully intersect the available imaging area. For example, a cone that only intersects one voxel on the edge of the image space can amplify noise in that voxel through the image reconstruction. To overcome the bias induced by cones or portions of cones outside of the imaging area, cones that intersect with less than $10 \%$ of the image space are removed. As the image space and the number of events increase during the dynamic measurement, the reconstruction speed decreases. In general search scenarios, the increase in the imaging space drives the reduction of the reconstruction speed, while in the 
mapping of highly contaminated areas, the number of recorded events drive the reduction in reconstruction speed. Thus, the approach presented here is limited in spatial size and number of counts.

Computationally, the gamma-ray image reconstruction runs on its own processing thread and uses the system location and 3-D model from SLAM along with the gamma-ray data as inputs. The $3-\mathrm{D}$ reconstruction returns results as it finishes processing and then recomputes the reconstruction. Thus its computation time span depends on the number of events and size of the model space.

\section{Results}

In the following section, the results of several measurements employing realtime 3D scene data fusion on a hand-portable gamma-ray imaging system are presented. Measurements of several source configurations with a range of source energies around and within objects are included.

\subsection{Single Source and 2-D Comparison}

In a lab environment, a Cs-137 source with an activity of $40 \mu \mathrm{Ci}$ is placed in the scene. The hand-portable HEMI was moved through the lab and the scene and the location of the source were reconstructed as the measurement progressed. Fig. 3 and Fig. 4 illustrates the concept of real-time scene and integrated gamma-ray image reconstruction by showing snapshots of the reconstruction and a visual image of where the detector and operator are in the scene.

The red line indicates the path of the instrument in the scene and the white dots indicate the locations of Compton events that were used for the gamma-ray reconstruction. The blue arrows indicate the scattering directions of the gamma rays for Compton events in the detector. The accuracy of the gamma-ray reconstruction is on the order of the voxel size of $10 \mathrm{~cm}$. This size is chosen to ensure the number of voxels does not get too large for the rooms measured in this paper. The measurement time was less than one minute. Some pixelization noise around the source is observed, which may result from the uncertainty 


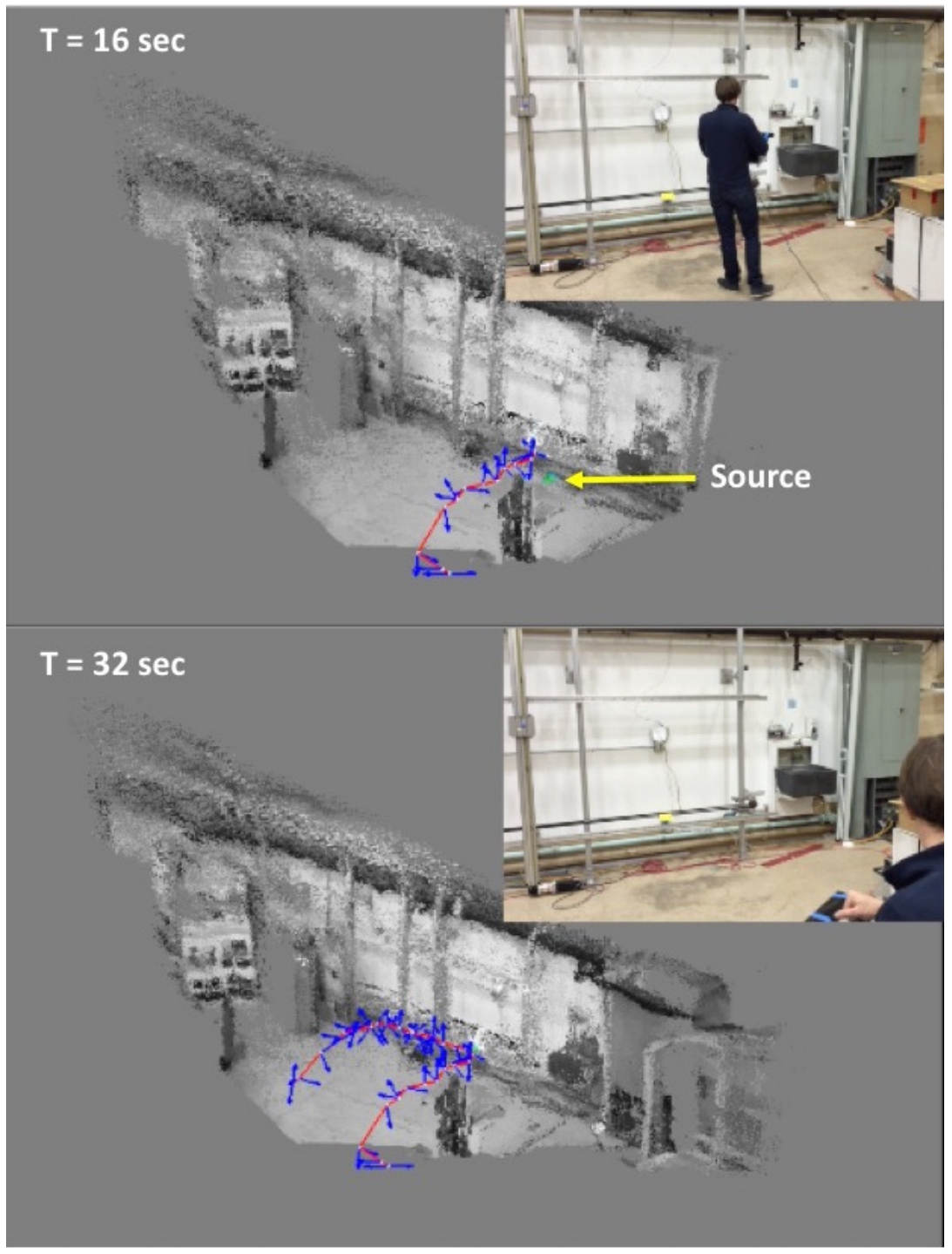

Figure 3: The evolution of the real-time reconstruction at 16 second intervals. The estimation for the Cs-137 source location improves as more data is collected. The blue arrows are the Compton events used in the reconstruction, the line is the path of the detector in the scene and the white circles are the location of the cone vertex. The details of the reconstruction are challenging to see in this figure, but it is shown to convey the fact that the result updates sequentially as more data is collected. 


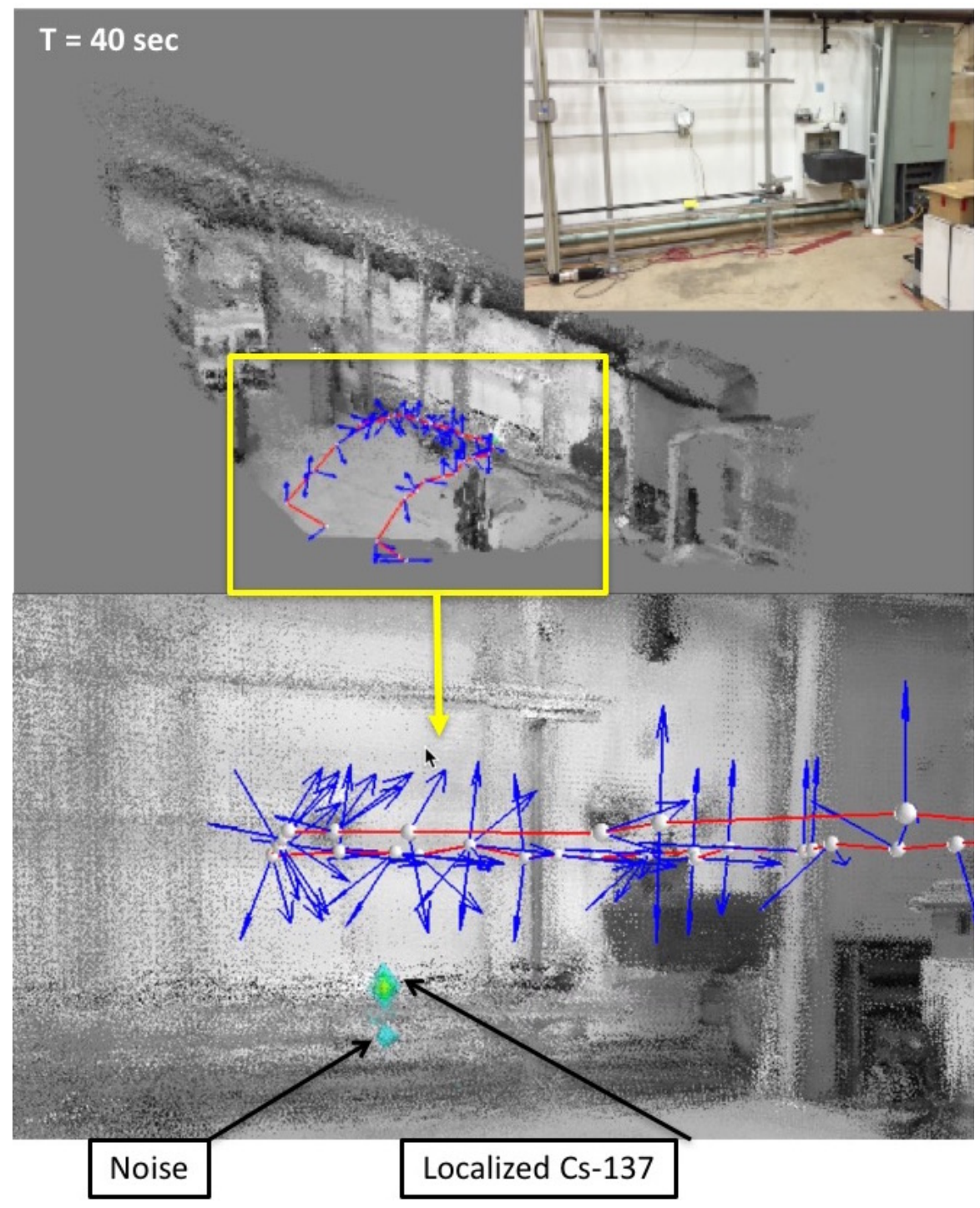

Figure 4: This shows the final reconstruction from walking around in a lab scene. The estimation for the Cs-137 source location improves as more data is collected. The blue arrows are the Compton events used in the reconstruction, the line is the path of the detector in the scene and the white circles are the location of the cone vertex. 
in the cone opening angle not reflecting the actual uncertainty inherent in the Compton cone. Subsequent measurements with a parameter for cone opening angle that better matches the angular resolution of the system (10 degrees) show a reduction in image noise, possibly as a result of a more realistic intersection of the probability surface with the voxels of the image space. In addition to the ability to reconstruct the location of a source in $3 \mathrm{D}$ with few events, the realtime feedback has the advantage of guiding the user and verifying the proper operation of the instrument and the reconstruction. For example, if the scene reconstruction or the tracking is lost, the user would quickly notice and can take appropriate action to return to a previous position and recover tracking.

In order to compare the 3-D method with conventional static 2-D imaging, HEMI is used as a static imager to reconstruct the 2-D projection of the gamma rays. For this measurement, HEMI was placed in the center of the walking path used to create the $3-\mathrm{D}$ reconstruction with the source in the same position, which puts HEMI $2.6 \mathrm{~m}$ from the source. At this distance, the spatial resolution at the sources is about $0.45 \mathrm{~m}$ FWHM given HEMI's angular resolution of 10 degrees FWHM. When HEMI is about $1 \mathrm{~m}$ away from the source, as is the case when walking around the room, the resolution is about $0.17 \mathrm{~m}$ FWHM. The given spatial resolution values are specific to this scenario, but it is important to note how the spatial resolution can be improved in the 3-D approach compared to the static 2-D approach. Fig. 5a shows the 2-D image that is created after $1 \mathrm{~min}$ measurement time, which is about the length of time for the 3-D measurement. The reconstruction method used is a real-time filtered back-projection (FBP) described in [16]. The result after one minute is noisy and inaccurate, with the hotspot off from the true location by several degrees. The image noise results from the low count rate and the fact that FBP does not include a proper Poisson noise model. The reconstructed image improves after further measurement time, as shown in Fig. 5b and the reconstructed source location is more accurate. This shows that, conceptually, the static 2-D approach provides less accurate information about the source location in a longer time. This is largely due to the inverse square of the distance fall off of the signal, a fundamental limit in 


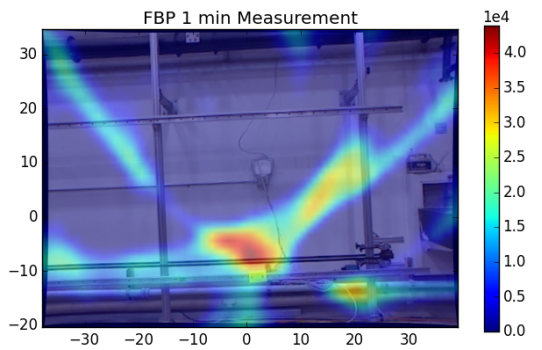

(a) 1 Minute, 58 Cones

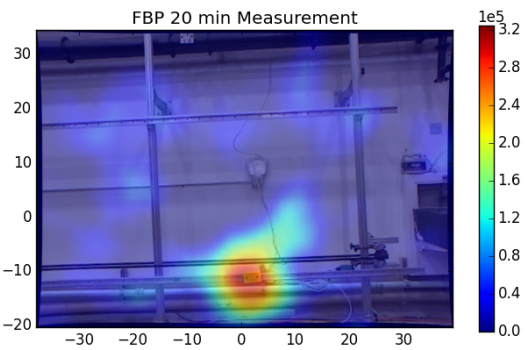

(b) 20 Minutes, 985 Cones

Figure 5: This shows a conventional representation of static 2-D Compton images of the Cs-137 source with a visual overlay of the lab scene.

the static mode and possibly that the 3 -D solution is constrained to the visual point cloud. Walking around the scene also helps improve spatial resolution when the detector moves closer to sources.

\subsection{Reconstruction of Multiple Sources}

One of the important features of scene data fusion from handheld HEMI is the ability to reconstruct gamma-ray images from a range of radioisotopes, each with different characteristic gamma-ray energies. To demonstrate this, three sources were placed on top of objects in a lab scene: Na-22, Cs-137 and Mn-54 with activities of $10 \mu \mathrm{Ci}, 8 \mu \mathrm{Ci}$, and $5 \mu \mathrm{Ci}$, respectively. Fig. 6 shows the coincidence energy spectrum obtained during a measurement, which is obtained by summing the energies from two interactions from a time coincident event. The coincidence time used was $1.2 \mu \mathrm{s}$ and was the same for all measurements in this work. All three sources can easily be identified in the spectra: Na-22 with energies of $511 \mathrm{keV}$ and $1275 \mathrm{keV}$, Cs-137 with $662 \mathrm{keV}$, and Mn-54 with $835 \mathrm{keV}$. Fig. 7 shows the reconstructed sources in the 3D scene obtained with energy gates set on $511 \mathrm{keV}$ (red), $662 \mathrm{keV}$ (green), and $835 \mathrm{keV}$ (blue) with a gate width of $20 \mathrm{keV}$. For each of these energies the source distribution was reconstructed over the entire volume encompassed by the visual data. All three sources were correctly localized within one voxel of the true source location. 


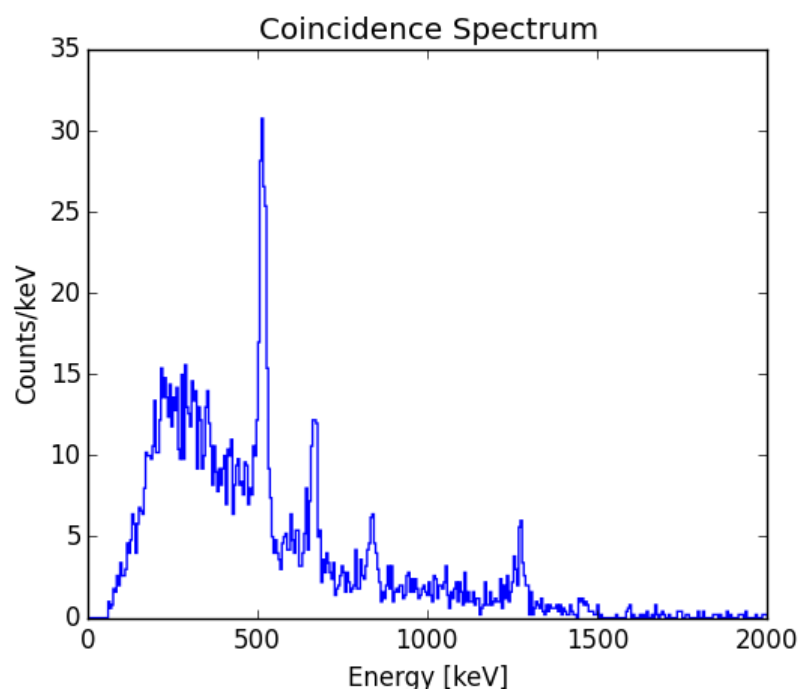

Figure 6: The coincident event energy spectra from a run with three sources, Na-22, Cs-137, and Mn-54. The total measurement time was 177 sec.

There is some broadening in the location of the source by several voxels, likely due to error in the tracking, which is illustrated by the variation in the white line that represents the path of the detector in the scene. This is due to accumulation of errors along the track and can be improved in future work.

Table 1 shows the parameters used in the measurement and reconstruction. The measurement time was less than 3 minutes. In this example, the imaging space is reduced by about a factor of 50 due to the constraints to the surfaces of the objects in the reconstructed scene. Table 2 shows some reconstruction time parameters, including the time to compute the system matrix for all the data and the time for 10 ML-EM iterations for the difference energy sources. This also shows the number of cones used in the reconstruction.

A second set of measurements was performed to illustrate the ability to integrate scene and gamma-ray image data for multiple sources contained in different containers or in cabinets. Ba-133, Na-22, and Cs-137 sources were hidden in a cupboard, a source safe, and a toolbox on top of a bench, respec- 


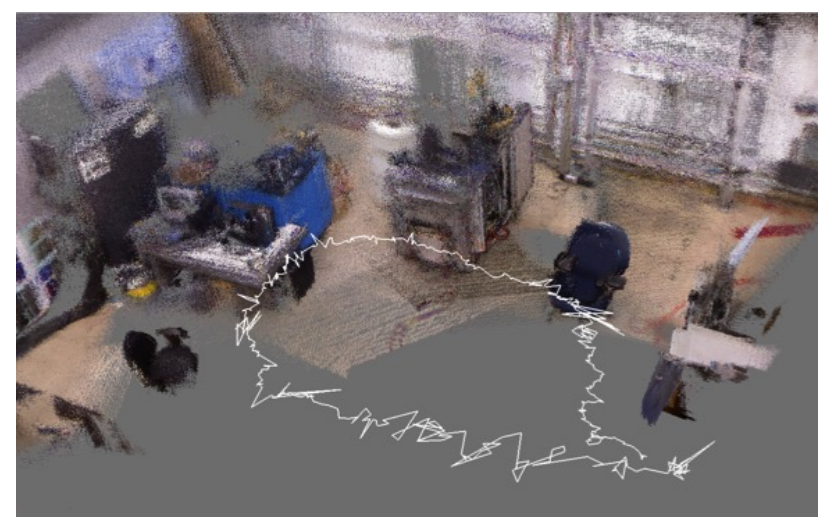

(a)

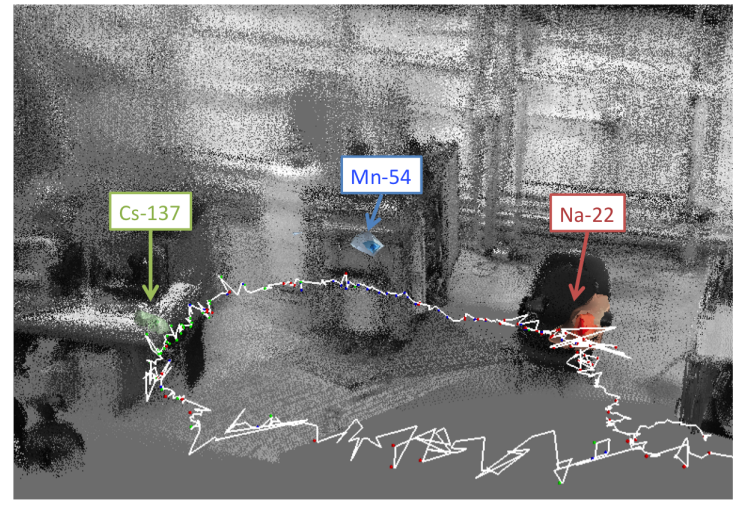

(b)

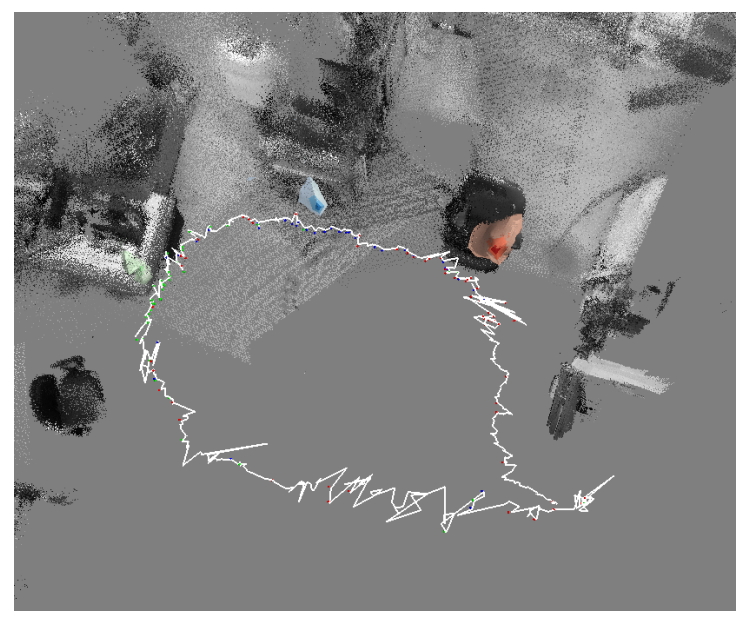

(c)

Figure 7: Figure (a) shows the 3-D colorized scene without the gamma-ray reconstruction. The white line reflects the path of the instrument in the scene. Figure (b) shows a side view of the scene with the reconstructed 3-D locations of three different gamma-ray source locations. In this figure the 3-D point cloud is plotted in black and white to allow better contrast with the colorized gammaray reconstructions. Figure (c) shows a top view of the scene with three sources visible. 


\begin{tabular}{|c|c|}
\hline Parameter & Value \\
\hline Voxel size & $10 \mathrm{~cm}$ \\
\hline Voxel Dimensions & $148 \times 226 \times 98$ \\
\hline Number of Iterations & 10 \\
\hline Measurement Time & $177 \mathrm{sec}$ \\
\hline Total Voxels & $3,277,904$ \\
\hline Restricted Voxels & 62,714 \\
\hline Percent Filled & $1.9 \%$ \\
\hline
\end{tabular}

Table 1: These are some parameters from the single source measurement. The voxel size represents the extension of a voxel in the image space. The voxel dimension represents the overall imaging space. The number of iterations refers to the iterative maximum-likelihood gamma-ray reconstruction. The restricted number of voxels refers to the number of voxels actually being used based on the scene reconstruction.

\begin{tabular}{|c|c|c|c|}
\hline Energy (keV) & Cones & System Matrix (ms) & 10 Iterations (ms) \\
\hline 511 & 233 & 924 & 112 \\
\hline 662 & 123 & 470 & 65 \\
\hline 834 & 67 & 266 & 37 \\
\hline
\end{tabular}

Table 2: This table shows some reconstruction time parameters for the three different sources shown in this section. 
tively, as shown in Fig. 8. The measurement time was about 100 seconds. The coincidence energy spectrum obtained in one measurement is shown in Fig. 9.

The energies at $511 \mathrm{keV}$ and $1275 \mathrm{keV}$ from Na-22 and the energy of $662 \mathrm{keV}$ from Cs-137 are clearly visible, while the strongest line of Ba-133 line at 356 $\mathrm{keV}$ has a poor signal-to-background and is barely visible. In spite of this, all three sources are localized. Fig. 8 shows the reconstructed scene and the fused gamma-ray image of the three source locations. In this reconstruction each color is a different energy as follows: red for $356 \mathrm{keV}$, green for $511 \mathrm{kev}$ and blue for $662 \mathrm{keV}$ corresponding to Ba-133, Na-22 and Cs-137 respectively. Some image noise is observed in the reconstruction of the photons at $356 \mathrm{keV}$ and $511 \mathrm{keV}$, which could be due to higher energy gamma-rays that are down-scattered into the detector or partial deposition escape events. It is interesting to note that the Ba-133 source is correctly localized even though the $356 \mathrm{keV}$ peak is not very prominent in the coincidence spectrum. The Cs-137 source inside the safe was localized slightly above the position inside the top of the safe. This could be due to restricting the reconstruction to the point cloud.

\subsection{Source Within an Object}

Scene data fusion with a handheld imager can also be used to position sources inside objects that are not accessible. To demonstrate this, a $30 \mu \mathrm{Ci}$ Cs-137 source was placed slightly off center inside of a cardboard box. Fig. 10 illustrates the ability to use the dynamic 3D imaging method to detect and localize sources inside of objects. The white lines are the tracked location of HEMI as it was moved in the scene. The red hotspot is the reconstructed source location that was obtained within a 50 second measurement time. In this reconstruction, the source was not restricted to the surface of the box. This increases the reconstruction time as the entire space around the track is voxelized to perform the reconstruction. In contrast to the previous examples, in this scenario it was possible to walk around the object of interest, thereby providing a more complete set of projections and making it reasonable to voxelize the entire space. 


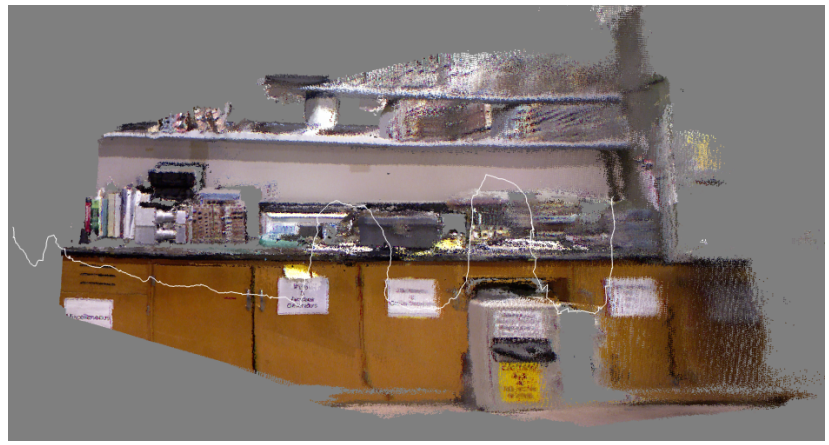

(a)

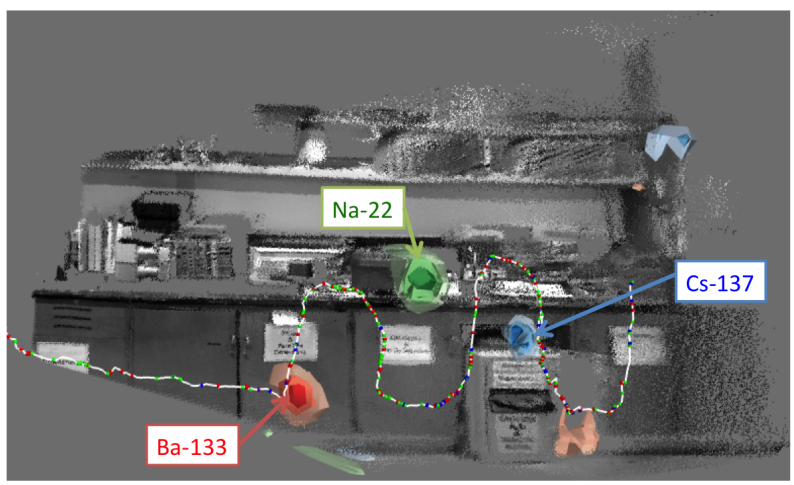

(b)

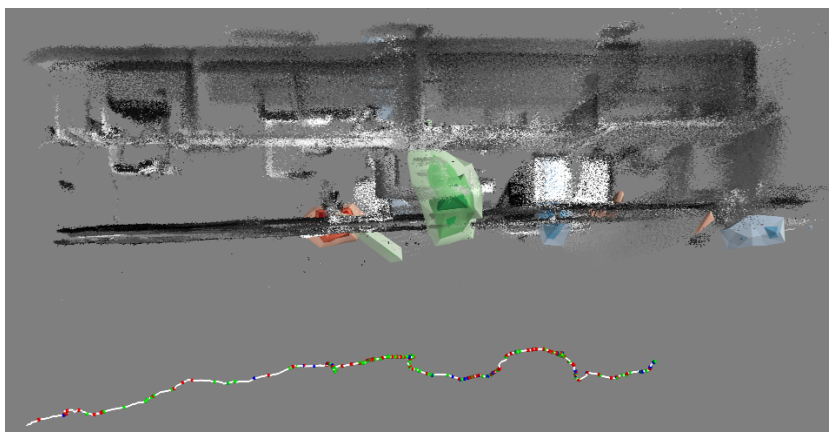

(c)

Figure 8: Figure (a) shows the 3-D colorized scene without the gamma-ray reconstruction. Figure (b) shows the three localized sources identified by their energies of $356 \mathrm{keV}$ (red), $511 \mathrm{keV}$ (green) and $662 \mathrm{keV}$ (blue) respectively. Figure (c) shows a top down view of the data shown in (b). The white line represents the path of the instrument relative to the scene. The colored dots on the path reflect gamma-ray events used in their respective image reconstruction. 


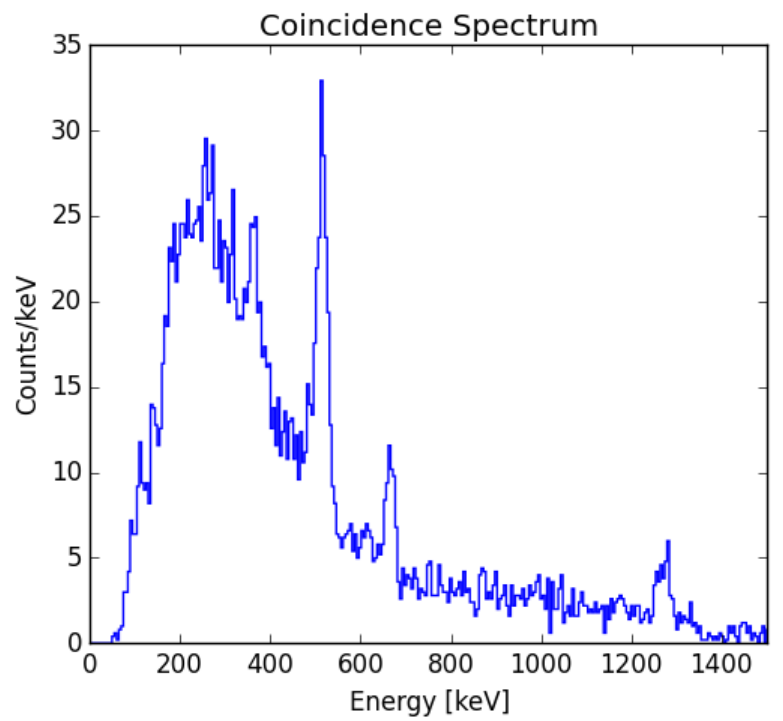

Figure 9: Coincidence energy spectrum from Ba-133, Na-22, and Cs-137 sources, placed in and around a lab bench as shown in Fig. 8. The $511 \mathrm{keV}$ and $1275 \mathrm{keV}$ lines of Na-22 and the $662 \mathrm{keV}$ line of Cs-137 are clearly visible. In contrast, the strongest line at $356 \mathrm{keV}$ of $\mathrm{Ba}-133$ is barely visible in the spectrum. The total measurement time was about 100 seconds. 


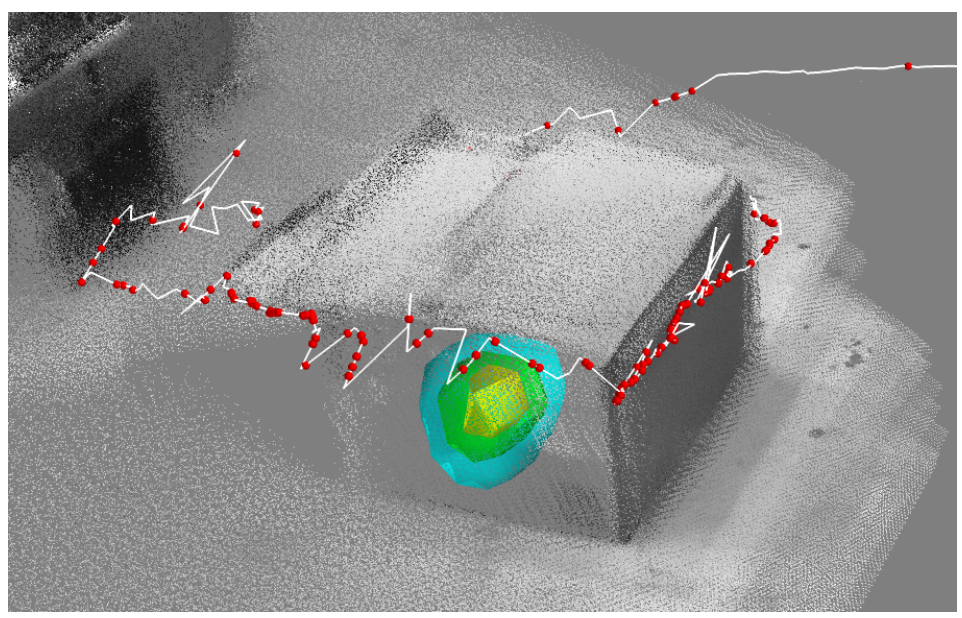

(a)

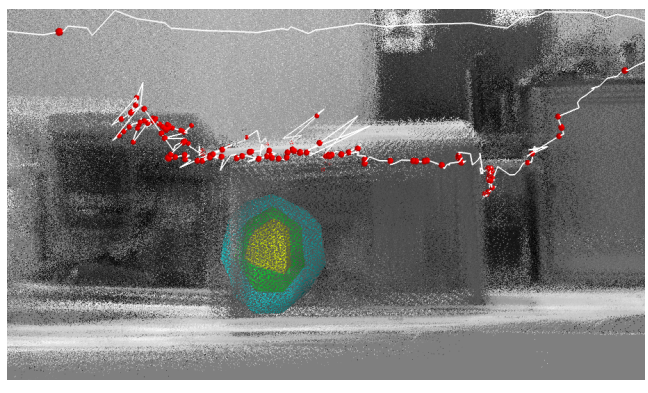

(b)

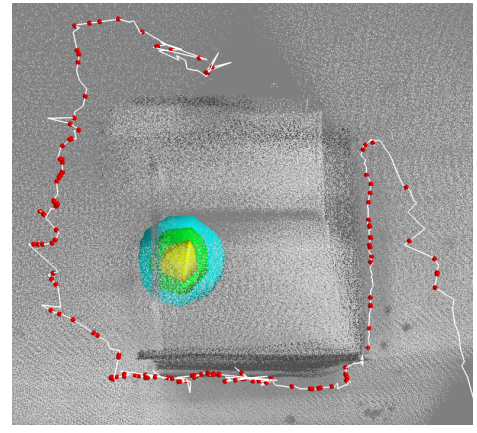

(c)

Figure 10: Reconstruction of a Cs-137 source inside of a box. The white line represents the path taken in the scene. The gamma-ray reconstruction was not constrained to any object. 


\section{Conclusion}

This paper demonstrates real-time fusion of gamma-ray image and visual scene data with a hand-held and mobile Compton imaging system. Due to the movement of the system, several projections are obtained enabling the 3 -D reconstruction of gamma-ray sources while at the same time increasing the speed and dimensionality of localization due to mitigating the $1 / \mathrm{r}^{2}$ intensity reduction. Measurements of sources placed openly in a lab and inside of a container were performed and localization was accomplished within several minutes for all cases, with sources on the order of $10 \mu \mathrm{Ci}$. 3-D localization is successfully demonstrated in room-sized lab environments, but further work is needed to extend this approach to larger scales while maintaining the $10 \mathrm{~cm}$ localization. While the Kinect is an affordable contextual sensor that can be integrated into the data acquisition and processing framework easily to provide real-time capabilities, it is limited to indoor scenarios due to its dependence on active infraredlight. However, visual cameras can be used to create 3-D models indoors and outdoors [17]. It is noteworthy that the mobile and real-time scene-data fusion concept is not restricted to portable gamma-ray imaging systems but can also be coupled with non-imaging counting or spectroscopic detectors. In addition, it can be extended to other modalities and radiation types, including, for example, the detection or imaging of neutrons.

\section{Acknowledgements}

We would like to thank Erika Suzuki for helping edit and prepare this manuscript. We acknowledge the support by the Lawrence Berkeley National Laboratory's LDRD program. This material is also based upon work supported by the Department of Energy National Nuclear Security Administration under Award number DE-NA0000979. This support does not constitute an express or implied endorsement on the part of the Government. 


\section{References}

[1] Christopher G. Wahl et al. "The Polaris-H imaging spectrometer". In: $\mathrm{Nu}$ clear Instruments and Methods in Physics Research Section A: Accelerators, Spectrometers, Detectors and Associated Equipment. Symposium on Radiation Measurements and Applications 2014 (SORMA XV) 784 (June 2015), pp. 377-381. ISSN: 0168-9002. DOI: $10.1016 /$ j . nima.2014.12.110.

[2] PHDS Co. - Germanium Gamma Ray Imaging Detectors. URL: http: //phdsco.com/.

[3] Shinichiro Takeda et al. "A portable Si/CdTe Compton camera and its applications to the visualization of radioactive substances". In: Nuclear Instruments and Methods in Physics Research Section A: Accelerators, Spectrometers, Detectors and Associated Equipment. New Developments in Photodetection NDIP14 787 (July 2015), pp. 207-211. ISSN: 0168-9002. DOI: $10.1016 / \mathrm{j} \cdot$ nima.2014.11.119.

[4] A. Kishimoto et al. "Demonstration of three-dimensional imaging based on handheld Compton camera". en. In: Journal of Instrumentation 10.11 (2015), P11001. ISSN: 1748-0221. DOI: 10 . 1088/1748-0221/10/11/ P11001.

[5] Klaus-Peter Ziock et al. A Mechanically-Cooled, Highly-Portable, HPGeBased, Coded-Aperture Gamma-Ray Imager. Tech. rep. Oak Ridge National Laboratory (ORNL), 2010.

[6] Lucian Mihailescu, Kai Vetter, and Daniel Chivers. "Standoff 3D gammaray imaging". In: Nuclear Science, IEEE Transactions on 56.2 (2009), pp. $479-486$.

[7] Ross Barnowski et al. "Scene data fusion: Real-time standoff volumetric gamma-ray imaging". In: Nuclear Instruments and Methods in Physics Research Section A: Accelerators, Spectrometers, Detectors and Associated Equipment (). ISSN: 0168-9002. DOI: 10.1016/j.nima.2015.08.016.

[8] A. Haefner et al. "3-D Aerial Gamma-ray Imaging”. In: In Preparation ().

[9] Yukihisa Sanada and Tatsuo Torii. "Aerial radiation monitoring around the Fukushima Dai-ichi nuclear power plant using an unmanned helicopter". In: Journal of Environmental Radioactivity 139 (Jan. 2015), pp. 294299. ISSN: 0265-931X. DOI: 10.1016/j.jenvrad.2014.06.027.

[10] Michelle Galloway et al. "Simulation and detector response for the High Efficiency Multimode Imager". In: Nuclear Instruments and Methods in Physics Research Section A: Accelerators, Spectrometers, Detectors and Associated Equipment. Symposium on Radiation Measurements and Applications (SORMA) XII 2010652.1 (Oct. 2011), pp. 641-645. ISSN: 01689002. DOI: $10.1016 / \mathrm{j}$.nima.2010.08.101.

[11] M. Amman et al. "Detector module development for the High Efficiency Multimode Imager". In: 2009 IEEE Nuclear Science Symposium Conference Record (NSS/MIC). Oct. 2009, pp. 981-985. DOI: 10.1109/NSSMIC. 2009.5402446 . 
[12] Michelle L Galloway. "Characterization and Applications of a CdZnTeBased Gamma-Ray Imager". PhD thesis. UC Berkeley, 2014.

[13] Felix Endres et al. "An evaluation of the RGB-D SLAM system". In: Robotics and Automation (ICRA), 2012 IEEE International Conference on. IEEE. 2012, pp. 1691-1696.

[14] L. Parra and H. H. Barrett. "List-mode likelihood: EM algorithm and image quality estimation demonstrated on 2-D PET". In: IEEE Transactions on Medical Imaging 17.2 (Apr. 1998), pp. 228-235. ISSN: 0278-0062. DOI: $10.1109 / 42.700734$.

[15] Donald W. Wilson, Benjamin MW Tsui, and Harrison H. Barrett. "Noise properties of the EM algorithm. II. Monte Carlo simulations". In: Physics in medicine and biology 39.5 (1994), p. 847.

[16] A. Haefner et al. "A Filtered Back-Projection Algorithm for 4 Compton Camera Data". In: IEEE Transactions on Nuclear Science 62.4 (Aug. 2015), pp. 1911-1917. ISSN: 0018-9499. DOI: 10.1109/TNS. 2015.2457436.

[17] Changchang Wu. "VisualSFM: A visual structure from motion system". In: URL: http://homes. cs. washington. edu/ ccwu/vsfm 9 (2011). URL: http://ccwu.me/vsfm/doc.html. 